
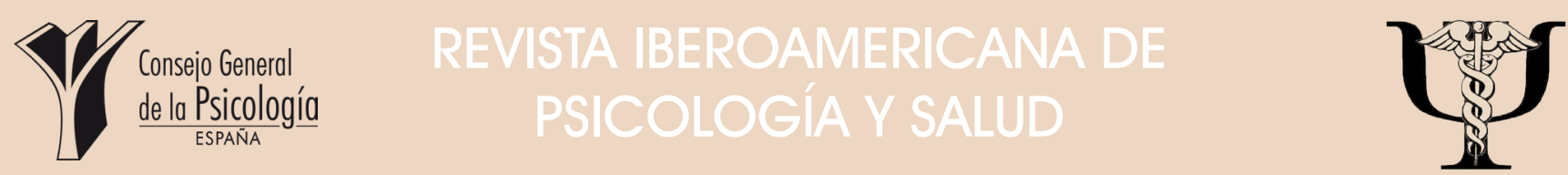

Revista Oficial de la Federación Iberoamericana de Asociaciones de Psicología (FIAP)

[Official Journal of the Latin-American Federation of Psychological Associations]

\title{
Violencia en el noviazgo, dependencia emocional y autoestima en adolescentes y jóvenes españoles
}

\author{
María de la Villa Moral ${ }^{*}$, Andrea García', Glenda Cuetos ${ }^{1}$ y Carlos Sirvent ${ }^{2}$
}

'Universidad de Oviedo (España).

${ }^{2}$ Fundación Instituto Spiral (España).

- Recibido: 7 - Febrero - 2017 . Aceptado: 11 - Abril - 2017 . Avance online: 12 - Mayo - 2017

RESUMEN. El objetivo de este estudio es comprobar las relaciones existentes entre Violencia en el Noviazgo, Dependencia Emocional y Autoestima en adolescentes y adultos jóvenes, así como las diferencias existentes en función del género y el nivel educativo. Mediante un muestreo bietápico de conveniencia con submuestreo aleatorio intragrupo se han seleccionado 224 adolescentes y adultos jóvenes con edades comprendidas entre los 15 y los 26 años $(M=18.2 ; D T=1.887)$ a los que se les aplicaron tres cuestionarios: Escala de Autoestima de Rosenberg, Inventario de Relaciones Interpersonales y Dependencias Sentimentales (IRIDS-100) y Cuestionario de Violencia entre Novios (CUVINO). De acuerdo con los resultados obtenidos, se ha hallado que los jóvenes victimizados presentan mayor dependencia emocional y menor autoestima que los no victimizados. En función del género, se encontró que las adolescentes víctimas de violencia tenían menor autoestima y los varones ejercían con más frecuencia procesos de acomodación y de manipulación. Se comprueba también una tasa superior de violencia y dependencia emocional en los estudiantes de Educación Secundaria frente a los universitarios. Se presentan las implicaciones de los resultados obtenidos tanto para investigaciones posteriores como para los programas de prevención e intervención y se señalan las fortalezas y limitaciones de este trabajo.

PALABRAS CLAVE: Violencia en el noviazgo, Dependencia emocional, Autoestima, Relaciones interpersonales, Adultos jóvenes, Género.

Dating violence, emotional dependence and self-esteem in spanish adolescents and young adults. ABSTRACT. The aim of this paper is to prove the existing links between Dating Violence, Emotional Dependence and Self-Esteem in adolescents and young adults and the existing differences depending on gender and educative level. 224 adolescents and young adults aged between 15 and 26 years old were analyzed $(M=18.2 ; S D=1.887)$ were selected using a two-sample sampling of convenience with intra-random random subsampling. Three questionnaires were applied to the students: Rosenberg Self-Esteem Scale, Interpersonal Relationships and Sentimental Dependences Inventory (IRIDS-100) and Dating Violence Questionnaire (CUVINO). Results point out that victimized young people show off more emotional dependence and lower self-esteem rates than non-victimized ones. Depending on gender, we found that victimized female have lower self-esteem while their male counterparts tend to use more accommodation and manipulation strategies. It also exists a higher rate of violence and emotional dependence in High School students than in University ones. The implications of the results for further research and for prevention and intervention programs are presented and limitations and strengths of the research are also pointed off.

KEYWORDS: Dating violence, Emotional dependence, Self-esteem, Interpersonal relations, Young adults Gender.

*Correspondencia: María de la Villa Moral Jiménez.

Facultad de Psicología, Plaza Feijóo, Universidad de Oviedo.

Código Postal: 33003, Oviedo, Asturias (España).

E-mail:mvilla@uniovi.es
En el amplio espectro de las relaciones de pareja, que abarca desde las relaciones de noviazgo recién iniciadas hasta los matrimonios consolidados, se manifiestan conductas violentas en mayor o menor medida (Batiza, 2017; Cala, Trigo y Saavedra, 2016; Medrano, Miranda 
y Figueras, 2017; Redding, Ruiz, Fernández. y Guijarro, 2017). La ubicación de esta problemática de forma exclusiva en el ámbito familiar, dándole connotaciones de privacidad, ha sido criticada (Aguilar, Alonso, Melgar y Molina, 2009; Amor y Echeburúa, 2010). Dada su naturaleza, también convendría replantearse la utilidad de la etiqueta violencia de género, pues forma parte de un sistema de relación violenta más amplio (Rodríguez-Franco, López-Cepero y Rodríguez-Díaz, 2010), además de no ser una categorización inclusiva. Específicamente, en este estudio se empleará el término "violencia en el noviazgo" (dating violence) para hacer referencia a aquellos comportamientos que, dentro de una relación íntima o de pareja, tienden a causar daño físico, psíquico y/o sexual a los miembros de una relación íntima (Celis y Rojas, 2015). Este comportamiento incluye agresiones físicas, relaciones sexuales forzadas y otras formas de coacción sexual, maltrato psicológico, diversos comportamientos dominantes e incluso la muerte, según Aiquipa (2015).

El estudio de esta problemática se ha centrado mayoritariamente en parejas adultas casadas priorizando el esquema de abusadorabusada, sin prestarle demasiada atención a las relaciones de noviazgo previas. Sin embargo, en los últimos años, se ha comprobado que la presencia de violencia en las relaciones de noviazgo de los adolescentes y adultos jóvenes es superior a la tasa de violencia perpetrada en las relaciones de convivencia estable, tanto en muestras anglosajonas (Jouriles, Garrido, Rosenfield y McDonald, 2009; Sears, Byers y Price, 2007) como específicamente en población española (Muñoz-Rivas, Graña, O’Leary y González, 2007; Rubio-Garay, LópezGonzález, Saúl y Sánchez-Elvira-Paniagua, 2012) y en jóvenes latinoamericanos (Celis y Rojas, 2015; Cortés-Ayala, Bringas-Molleda, Rodríguez-Franco, Flores-Galaz, Ramiro y Rodríguez-Díaz, 2014; Cortés-Ayala, Flores, Bringas, Rodríguez-Franco, López-Cepero y Rodríguez-Díaz, 2015; Rey-Anacona, 2013; ReyAnacona, Mateus-Cubides y Bayona-Arévalo, 2010). De hecho, aunque los resultados varíen sensiblemente dependiendo de los instrumentos de investigación y de la definición de violencia empleada, se encuentran tasas de prevalencia de violencia dos o tres veces superiores a las halladas cuando se estudian muestras de parejas adultas (González-Ortega, Echeburúa y Corral, 2008; Póo y Vizcarra, 2008).

Los estudios sobre violencia en parejas de adolescentes y adultos jóvenes han revelado una serie de características propias, no aplicables a parejas casadas (López-Cepero, RodríguezFranco, Rodríguez-Díaz, Bringas y Paíno, 2015; Rodríguez-Franco et al., 2016). Según Rey-Anacona (2008), los motivos por los que se continúa con la relación, a pesar de las agresiones, son diferentes a los que mantienen las relaciones en los casos de violencia conyugal, ya que no existen de por medio responsabilidades parentales, contractuales $\mathrm{O}$ dependencia económica entre los miembros. De acuerdo con González-Ortega et al. (2008) algunos factores como: inmadurez emocional, expectativas idealizadas del amor, sesgos cognitivos, presencia de actitudes y creencias conservadoras sobre los roles de género y modelos sexistas que disculpen la violencia pueden influir en que se mantengan las relaciones de noviazgo conflictuadas. También es necesario tener en cuenta que en muchas ocasiones, las víctimas no interpretan las agresiones como tal, sino que las trivializan (Jouriles et al., 2009). En este sentido, resulta de interés contrastar las percepciones sobre violencia sutil y manifiesta. Así, Novo, Herbón y Amado (2016) confirmaron una baja detección de la violencia sutil como constitutiva de violencia de género y concluyeron que los contextos de violencia manifiesta son evaluados como más representativos de violencia de género que los sutiles, habiéndose hallado que los varones valoraron que la violencia sutil influye más en el comportamiento de la víctima y la manifiesta en que la víctima acceda a las peticiones de su pareja, tendencia contraria a la hallada en las mujeres.

La reciprocidad de la violencia en las relaciones de noviazgo ha sido suficientemente constatada (Alegría y Rodríguez, 2015; González-Ortega et al., 2008) y se ha asociado a una distribución de poder entre ambos sexos más igualitaria en los adolescentes y los adultos jóvenes (Póo y Vizcarra, 2008). Incluso se apunta a que las chicas perpetran e inician la violencia con más frecuencia que los chicos (Celis y Rojas; 2015; Cortés-Ayala et al., 2015; Pradas y Perles, 2012) excepto en el caso de las agresiones físicas severas, en 
las que la tendencia se invierte (Muñoz-Rivas et al., 2007). Respecto al trasfondo psicosocial interpretativo, en estudios como el de Cuadrado (2012) se concluye que los chicos interpretan muchas de las modalidades de maltrato como mecanismos de interacción social, aunque las chicas los atribuyen a la intencionalidad de dañar y a mecanismos de desequilibrio de poder. En otro orden, la asimilación de los tópicos del amor romántico se asocia a patrones relacionales desadaptativos, lo cual se vincula a la identificación de la pareja con el ideal romántico, los pensamientos intrusivos, el proceso inhabilitante de idealización del otro y de la relación y la creencia en algo "mágico" en la relación, entre otros, como principales signos de tal proceso de retroalimentación (Moral et al., 2016).

Tales conductas desadaptativas pueden derivar en que la necesidad de afecto se convierta en algo patológico, excediendo las necesidades de vinculación propias de cada ser humano y derivando en Dependencia Emocional (DE). Nuestra concepción de la DE como una adicción conlleva la necesidad de contextualizarla en un marco conceptual más amplio, el de las Dependencias Sentimentales - Afectivas, caracterizadas por la manifestación de comportamientos adictivos en una relación interpersonal con asimetría de rol y una actitud dependiente en relación al otro/a (Moral y Sirvent, 2008). En ese sentido, podría definirse la DE como "un patrón crónico de demandas afectivas frustradas, que buscan desesperadamente satisfacerse mediante relaciones interpersonales de apego patológico" (Moral y Sirvent, 2009, p. $231)$ y cuyos descriptores clínicos y psicosociales son la posesividad, el desgaste energético intenso, la persistencia en la vinculación, la voracidad de cariño, los sentimientos negativos y la preferencia por relaciones asimétricas en las que adoptar una posición subordinada. Específicamente, autoras como Hirigoyen (2006) afirman que la DE es una consecuencia del dominio y la manipulación de una pareja violenta, ya que estas crean una verdadera adicción que se puede explicar por mecanismos neurobiológicos y psicológicos que intentan, sin éxito, disminuir el sufrimiento del miembro de la pareja que sufre los abusos.

La dependencia emocional no es una problemática exclusiva de un determinado grupo de individuos, por lo que se manifiesta en diferentes rangos de edad. En este sentido, Rodríguez-Franco, Rodríguez-Díaz, AntuñaBellerín y López-Cepero (2010) señalan que esta problemática aparece con frecuencia en la etapa del ciclo vital en el que se inician las relaciones de pareja (adolescencia tardía y juventud temprana). En este rango de edad se considera importante la entrega incondicional al otro y con frecuencia ambos sexos consideran tanto los aspectos placenteros como dolorosos de las relaciones, lo cual podría hacerles depender emocionalmente de sus parejas (Pradas y Perles, 2012). Respecto a las diferencias intergénero, dados los roles sociales diferenciales, los varones expresan su dependencia de forma larvada, de modo que coexisten en el sujeto la necesidad afectiva y una intensa agresividad en la relación, es decir, el dependiente emocional no solo necesita, sino que también menosprecia a su pareja (Sirvent, 2000). En este sentido, se ha constatado una tendencia a la búsqueda de sensaciones y a la hiperestimulación por parte de los varones dependientes emocionales, mientras que las mujeres recurren con más frecuencia al autoengaño y al uso de mecanismos de negación y no afrontamiento (Moral y Sirvent, 2009). En este contexto, el autoengaño es un constructo que se caracteriza por engañarse a uno mismo derivando en un comportamiento regido por mecanismos de acción, tales como insinceridad y opacidad comunicativa, mixtificación y mecanismos de manipulación y reincidencia, entre otros, y que induce a un registro de la realidad distorsionado (Sirvent, 2006). Representa la incapacidad del sujeto para darse cuenta de los efectos adversos de la relación, de manera que en dependientes emocionales se asocia a mecanismos de negación a modo de rechazo, reprobación y no reconocimiento de una relación conflictuada y que conduce a una situación de no afrontamiento (Moral et al., 2016).

Vinculando el estudio de la autoestima a la violencia y a la dependencia emocional en las relaciones de pareja se constata que la baja autoestima ha sido mencionada en múltiples ocasiones como una consecuencia de la violencia en el noviazgo (González-Ortega et al., 2008; Póo y Vizcarra, 2008; Rey-Anacona, 2008, 2013) y también como una dificultad adicional para salir de tales relaciones, al 
justificar la víctima las agresiones (Amor, Bohórquez y Echeburúa, 2006). Además, en los dependientes emocionales su autoestima puede verse mermada al estar inmersos en una relación afectivodependiente con personas por lo general narcisistas y explotadoras (Sirvent, 2000). Por otra parte, autores como Pradas y Perles (2012) y Rodríguez-Franco et al. (2010) señalan que la DE estaría en la raíz del mantenimiento de las relaciones de noviazgo violentas, ya que, al igual que la autoestima, aumenta la tolerancia a los abusos recibidos y dificulta que se termine con la relación. En este sentido, Amor et al. (2006) consideran que la yuxtaposición temporal entre el buen y el mal trato por parte del agresor provoca, por una parte, bajadas en la autoestima y por otra, una necesidad incrementada de la pareja, lo que da lugar a una situación conocida como apego paradóiico (véase Aiquipa, 2015).

Teniendo en cuenta lo expuesto anteriormente se puede afirmar que existe una relación bien establecida entre la dependencia emocional, la autoestima y la violencia en el noviazgo. El estudio de dicha relación en los adolescentes resulta de sumo interés, de modo que la propia experiencia en este tipo de relaciones problemáticas facilitará la adopción de actitudes inadecuadas en relaciones posteriores (Rodríguez-Franco, López-Cepero, RodríguezDíaz y Antuña-Bellerín, 2009), además de las posibles consecuencias a nivel psicosocial y socosanitario (Amor et al., 2006). Por todo ello, y teniendo en cuenta que la violencia en las parejas jóvenes puede actuar como un precursor de la violencia en las parejas adultas, se hace necesario estudiar este fenómeno, centrándonos en la vinculación entre DE y autoestima.

Específicamente, el objetivo principal de este estudio es comprobar la relación existente entre violencia en el noviazgo, dependencia emocional y autoestima, planteándose el estudio de las diferencias inter-género y en función del nivel educativo en los indicadores citados.

Como objetivos específicos se proponen los siguientes:

a) Explorar las diferencias en los indicadores de dependencia emocional y en autoestima entre el grupo de víctimas de violencia en el noviazgo y el grupo sobre el que no se ha ejercido violencia.

b) Analizar las diferencias inter-género entre los adolescentes victimizados en dependencia emocional, con especial interés en los indicadores de autoengaño y en los mecanismos de negación y no afrontamiento, y en autoestima.

c) Evaluar la existencia de diferencias significativas en las experiencias de victimización, así como en dependencia emocional y autoestima en función del nivel educativo (Universitario o Preuniversitario).

\section{MÉTODO}

\section{- PARTICIPANTES Y DISEÑO}

En este estudio han participado 226 estudiantes de enseñanzas preuniversitarias $\left(4^{\circ}\right.$ de Educación Secundaria y $1^{\circ}$ y $2^{\circ}$ de Bachillerato) y universitarias $\left(1^{\circ}\right.$ y $2^{\circ}$ de Logopedia y $1^{\circ}$ de Psicología) cuya edad oscilaba entre 15 y 26 años, siendo la media de edad de 18.2 años $(D T=1.887)$. Han participado 168 chicas (74.3\%) y 58 chicos (25.7\%), de modo que la distribución desigual de géneros se debe a que las carreras universitarias seleccionadas son cursadas mayoritariamente por mujeres. Para la selección de los participantes se ha llevado a cabo un muestreo bietápico con muestreo no probabilístico de tipo incidental en la Facultad de Psicología y en el I.E.S. "Aramo", ambos de Oviedo (España), con submuestreo por conglomerados y aleatorio intragrupo.

\section{- INSTRUMENTOS Y VARIABLES}

Para evaluar la autoestima se ha empleado la Escala de autoestima de Rosenberg adaptada al español por Echeburúa (), que tiene como objetivo evaluar el grado de satisfacción que presenta una persona consigo misma. Consta de 10 ítems generales que puntúan de 1 a 4 en una escala tipo Likert, estando fijado en la puntuación de 29 el punto de corte. El coeficiente alfa de consistencia interna es de 0.92 (Sarasua, Zubizarreta, Echeburúa y Corral, 2007), habiéndose obtenido en este estudio un valor de 0.918 .

El Inventario de Relaciones Interpersonales y Dependencias Sentimentales (IRIDS-100) de Sirvent y Moral (2005) es un instrumento de medida para la evaluación de las dependencias afectivas que está compuesto por 100 ítems evaluados mediante una escala Likert de 5 puntos evaluados desde "Muy de acuerdo" a 
"Muy en Desacuedo". La estructura factorial del inventario consta de 7 dimensiones y 23 factores sindrómicos. El alfa global de la escala es de 0.985 (Sirvent y Moral, 2005), siendo el valor obtenido en este estudio de 0.927 y oscilando entre el obtenido en el factor Sentimientos Negativos $(\alpha=0.916)$ y en Acomodación $(\alpha=0.794)$. Teniendo en cuenta que para el propósito de esta investigación las dependencias relacionales mediatizadas carecían de interés, se decidió suprimir la subescala de Bidependencia. Asimismo, se suprimieron las dimensiones de Identidad/Caracterosis (con sus tres factores: identidad/identificación, límites débiles y límites rígidos) y de Tríada Codependiente (con los factores orientación rescatadora y sobrecontrol).

El Cuestionario de Violencia entre Novios (CUVINO) es un instrumento de evaluación de la victimización en las relaciones de noviazgo dirigido específicamente a adolescentes y jóvenes, validado en jóvenes hispanohablantes por Rodríguez-Franco, López-Cepero, RodríguezDíaz, Bringas, Antuña y Estrada (2010). Se compone de 42 indicadores evaluados en una escala Likert de 5 puntos (de 0 a 4 ) agrupados en ocho factores: Desapego, Humillación, Sexual, Coerción, Físico, Género, Castigo Emocional e Instrumental y tres etiquetas de tipo dicotómico (Maltrato, Miedo y Atrapamiento) que ofrecen ocho combinaciones de respuesta posibles. La fiabilidad total de la escala es de $\alpha=0.935$ (Rodríguez-Franco et al., 2010) con un rango de amplitud de fiabilidad de 0.818 (Humillación) a 0.558 (Castigo Instrumental). Específicamente, en este estudio se ha hallado una fiabilidad total de la escala de 0.897 con valores que oscilan entre 0.803 en Humillación y 0.540 en Castigo Instrumental.

\section{- PROCEDIMIENTO Y ANÁLISIS DE DATOS}

La recogida de datos se llevó a cabo mediante una aplicación colectiva de los cuestionarios en cada una de las aulas evaluadas. Para ello, se solicitó la participación voluntaria de los alumnos que firmaron un acuerdo de consentimiento informado y se les garantizó en todo momento su anonimato y el uso exclusivo de sus respuestas para fines de investigación. Miembros del equipo e investigación entrenados al efecto les proporcionaron las instrucciones necesarias para cubrir los cuestionarios y se mostró disponibilidad para resolver cualquier duda. Durante la aplicación en la Universidad y Bachillerato estuvo presente el profesor de la asignatura impartida a esa hora y en el caso de la ESO, el tutor.

Una vez recogidos los datos, se procedió a analizarlos estadísticamente con el SPSS versión 20. En el análisis se incluyeron los estadísticos descriptivos para la muestra presentados en los apartados anteriores, pruebas para verificar la distribución de normalidad de las variables dependientes y las pruebas $T$ de Student y $U$ de Mann-Whitney para comparar las puntuaciones intergrupales.

\section{RESULTADOS}

En primer lugar se exploró la frecuencia de victimización en base a dos criterios distintos: la percepción del sujeto sobre su experiencia de victimización (representado por la etiqueta "maltrato" del CUVINO) y un criterio de tolerancia cero (basado en las respuestas a los ítems conductuales de dicho cuestionario; al utilizar un criterio de tolerancia cero se considera que cualquier sujeto que responda afirmativamente a, por lo menos un ítem, habrá sido victimizado). En la tabla 1 se muestran los estadísticos descriptivos hallados al comparar ambos criterios. Se comprueba que existen diferencias entre la percepción de victimización de los sujetos y la clasificación obtenida empleando el criterio de tolerancia cero. Por ello, y siguiendo las perspectivas teóricas que señalan que las víctimas de violencia en la pareja no siempre son capaces de identificarse como tal, emplearemos el criterio de tolerancia cero para dividir la muestra para los análisis posteriores.

\begin{tabular}{|l|c|c|c|c|}
\hline \multicolumn{5}{|c|}{$\begin{array}{c}\text { Tabla } 7 \\
\text { Frecuencia de victimización por género en función del criterio } \\
\text { observado }\end{array}$} \\
\hline \multirow{2}{*}{ Criterio } & Sexo & Victimización & Frecuencia & $\%$ \\
\hline Tolerancia cero & Hombre & Sí & 53 & $91.4 \%$ \\
& & No & 5 & $8.6 \%$ \\
& Mujer & Sí & 137 & $82.5 \%$ \\
& & No & 29 & $17.5 \%$ \\
Etiqueta & Hombre & Sí & 2 & $3.4 \%$ \\
Maltrato & & No & 56 & $96.6 \%$ \\
& & Sí & 14 & $8.4 \%$ \\
& Mujer & No & 152 & $91.6 \%$ \\
\hline
\end{tabular}


Se ha empleado la prueba K-S para comprobar el ajuste a la curva normal de las subescalas y factores contemplados en el cuestionario IRIDS-100, así como el nivel de significación de los mismos. En nuestro estudio, únicamente las escalas de Dependencia Emocional $(p=.104)$ y Codependencia ( $p=$ .509), se ajustan a una distribución normal comprobándose en ambos casos la significación de las diferencias $(t=7.032, t=7.848 ; p<.000)$ y con un tamaño del efecto medio (>.50). El empleo de la estadística no paramétrica se justifica ya que en este estudio la distribución muestral subyacente no se ajusta a los criterios paramétricos, de modo que no se puede asumir que los datos se ajusten a una distribución conocida, si bien en todos los indicadores se cumple el criterio de homocedasticidad. La posible reducción de eficiencia es compensada por un elevado tamaño muestral.
Los resultados de la prueba $U$ de MannWhitney para los diversos factores que exploran Dependencia Emocional y para el indicador de Autoestima, en función de la presencia o no de una experiencia de victimización, se muestran en la Tabla 2. Se observa que las diferencias en todos los factores analizados resultan significativas $(p<.05)$, si bien teniendo en cuenta el estadístico $r$ el tamaño del efecto es pequeño para todos los factores analizados. Se confirma la existencia de diferencias estadísticamente significativas en Dependencia Emocional y Autoestima entre los adolescentes que han sido víctimas de violencia en su relación de pareja y los que no. Además, los resultados apuntan a una autoestima más baja y a una mayor gravedad de los síntomas de dependencia emocional en aquellos adolescentes que han sido victimizados.

Tabla 2

Diferencias en los factores de Dependencia Emocional y en Autoestima en función de la percepción de Victimización (prueba 4 )

\begin{tabular}{|c|c|c|c|c|c|}
\hline \multirow{3}{*}{ Factores } & \multicolumn{2}{|c|}{ Tipo de Muestra } & \multirow{3}{*}{$\boldsymbol{U}$} & \multirow{3}{*}{$p$} & \multirow{3}{*}{$\boldsymbol{r}$} \\
\hline & Sin violencia $(n=34)$ & Con Violencia $(n=190)$ & & & \\
\hline & 0 & $\boldsymbol{R P}$ & & & \\
\hline Dependencia pura & 146.49 & 106.42 & 2074.5 & $.001 *$ & .222 \\
\hline Búsqueda de sensaciones & 157.09 & 104.52 & 1714.0 & $.000^{*}$ & .291 \\
\hline Craving / Abstinencia & 138.94 & 107.77 & 2331.0 & $.009^{*}$ & .173 \\
\hline Acomodación & 137.22 & 108.08 & 2389.5 & $.015^{\star \star}$ & .162 \\
\hline Autoengaño & 154.56 & 104.97 & 1800.0 & $.000^{*}$ & .275 \\
\hline Manipulación & 144.25 & 106.82 & 2150.5 & $.002^{*}$ & .210 \\
\hline Reiteración & 145.16 & 106.66 & 2119.5 & $.001 *$ & .214 \\
\hline $\begin{array}{l}\text { Mecanismos de negación y no } \\
\text { afrontamiento }\end{array}$ & 142.81 & 107.08 & 2199.5 & $.003^{*}$ & .199 \\
\hline Soledad & 133.40 & 108.76 & 2519.5 & $.038^{* *}$ & .139 \\
\hline Vacío emocional & 147.74 & 106.19 & 2032.0 & $.001^{*}$ & .231 \\
\hline Culpa & 153.25 & 105.21 & 1844.5 & $.000^{*}$ & .268 \\
\hline Autodestrucción & 153.74 & 105.12 & 1828.0 & $.000^{*}$ & .273 \\
\hline Inescapabilidad emocional & 156.85 & 104.56 & 1722.0 & $.000^{*}$ & .291 \\
\hline $\begin{array}{l}\text { Recreación de sentimientos } \\
\text { negativos }\end{array}$ & 142.06 & 107.21 & 2225.0 & $.003^{*}$ & 197 \\
\hline Antecedentes personales & 138.60 & 107.83 & 2342.5 & $.010^{*}$ & .172 \\
\hline Focalización en el otro & 155.69 & 104.77 & 1671.5 & $.000^{*}$ & .283 \\
\hline Autoestima & 130.62 & 109.26 & 2614.0 & $.018^{* *}$ & .158 \\
\hline
\end{tabular}


Por su parte, en la Tabla 3 se muestran los resultados de la prueba $T$ para las diferencias inter-género entre los adolescentes victimizados en las variables Autoestima y Dependencia Emocional. Puede observarse la existencia de diferencias estadísticamente significativas únicamente en la variable Autoestima $(p=.006)$, con un tamaño del efecto medio $(d=.471)$. Este hallazgo señala que no existen diferencias intergénero en cuanto a la $D E$, pero estas sí aparecen en la autoestima, siendo esta significativamente más baja en el caso de las mujeres.

Diferencias inter-género en Dependencia Emocional y en Autoestima (prueba T)

\begin{tabular}{|c|c|c|c|c|c|c|c|}
\hline \multirow{3}{*}{ Factores } & \multicolumn{4}{|c|}{ Tipo de Muestra } & \multirow{3}{*}{$\boldsymbol{T}$} & \multirow{3}{*}{$\boldsymbol{p}$} & \multirow{3}{*}{$d$} \\
\hline & \multicolumn{2}{|c|}{ Hombre $(n=53)$} & \multicolumn{2}{|c|}{ Mujer $(n=137)$} & & & \\
\hline & $M$ & $D T$ & $M$ & DT & & & \\
\hline $\begin{array}{l}\text { Dependencia } \\
\text { Emocional }\end{array}$ & 3.753 & 0.642 & 3.742 & 0.651 & 0.103 & .918 & - \\
\hline Autoestima & 1.849 & 0.316 & 1.672 & 0.471 & 2.777 & .006 & .471 \\
\hline
\end{tabular}

Los resultados de la prueba $U$ de MannWhitney para las restantes subescalas, dimensiones y factores del IRIDS-100 en función del género de las víctimas se presentan en la Tabla 4. Se aprecian diferencias estadísticamente significativas en los factores Acomodación $(p<.001)$ y Manipulación $(p=.005)$.
El tamaño del efecto para las variables estadísticamente significativas es medio $(r=.272$ y $r=.204$, respectivamente). Dichas diferencias señalan que los varones victimizados presentan una mayor acomodación y manipulación que las mujeres.

Tabla 4

Diferencias inter-género en los factores de Dependencia Emocional (prueba $U$

\begin{tabular}{|c|c|c|c|c|c|}
\hline \multirow{3}{*}{ Factores } & \multicolumn{2}{|c|}{ Tipo de Muestra } & \multirow{3}{*}{$\boldsymbol{U}$} & \multirow{3}{*}{$\boldsymbol{p}$} & \multirow{3}{*}{$\boldsymbol{r}$} \\
\hline & Hombre $(n=53)$ & Mujer $(n=137)$ & & & \\
\hline & $\boldsymbol{R P}$ & $\boldsymbol{R P}$ & & & \\
\hline Dependencia pura & 90.82 & 97.31 & 3382.5 & .465 & - \\
\hline Búsqueda de sensaciones & 93.75 & 96.18 & 3538.0 & .785 & - \\
\hline Craving / Abstinencia & 98.86 & 94.20 & 3452.5 & .599 & - \\
\hline Acomodación & 71.58 & 104.76 & 2362.5 & $.000^{*}$ & .272 \\
\hline Autoengaño & 91.80 & 96.93 & 3434.5 & .564 & - \\
\hline Manipulación & 77.65 & 102.41 & 2684.5 & $.005^{\star}$ & .204 \\
\hline Reiteración & 98.98 & 94.15 & 3446.0 & .586 & - \\
\hline $\begin{array}{l}\text { Mecanismos de negación y no } \\
\text { afrontamiento }\end{array}$ & 100.99 & 93.38 & 3339.5 & .390 & - \\
\hline Soledad & 88.99 & 98.08 & 3285.5 & .304 & - \\
\hline Vacío emocional & 96.61 & 95.07 & 3571.5 & .862 & - \\
\hline Culpa & 93.21 & 96.39 & 3509.0 & .719 & - \\
\hline Autodestrucción & 96.12 & 95.26 & 3597.5 & .922 & - \\
\hline Inescapabilidad emocional & 91.21 & 97.16 & 3403.0 & .502 & - \\
\hline $\begin{array}{l}\text { Recreación de sentimientos } \\
\text { negativos }\end{array}$ & 103.87 & 92.26 & 3187.0 & .185 & - \\
\hline Antecedentes personales & 88.75 & 98.11 & 3272.5 & .288 & - \\
\hline Focalización en el otro & 86.81 & 98.86 & 3170.0 & .174 & - \\
\hline
\end{tabular}


Por último, en la Tabla 5 se exponen los resultados de la prueba $T$ para la comparación de la subescala Dependencia Emocional (IRIDS-1), Autoestima y una medida del grado de victimización en función del nivel educativo (Universitario o Preuniversitario). Se puede observar la existencia de diferencias estadísticamente significativas en el caso de la subescala Dependencia Emocional ( $p=.003)$ y el grado de Victimización $(p=.019)$, con un tamaño del efecto moderado (con valores de .430 y .347 , respectivamente). En virtud de estas diferencias se comprueba que la victimización es más intensa en los preuniversitarios y que además presentan una mayor dependencia emocional.

Tabla 5

Diferencias en Dependencia Emocional, Autoestima y Victimización en función del nivel académico (prueba T)

\begin{tabular}{|c|c|c|c|c|c|c|c|}
\hline \multirow{3}{*}{ Factores } & \multicolumn{4}{|c|}{ Tipo de Muestra } & \multirow{3}{*}{$\boldsymbol{T}$} & \multirow{3}{*}{$p$} & \multirow{3}{*}{$d$} \\
\hline & \multicolumn{2}{|c|}{ Preuniversitario $(n=87)$} & \multicolumn{2}{|c|}{ Universitario $(n=103)$} & & & \\
\hline & $\boldsymbol{M}$ & $D T$ & $\boldsymbol{M}$ & DT & & & \\
\hline $\begin{array}{l}\text { Dependencia } \\
\text { Emocional }\end{array}$ & 3.597 & 0.640 & 3.870 & 0.629 & -2.960 & $.003^{*}$ & .430 \\
\hline Autoestima & 1.724 & 0.445 & 1.718 & 0.452 & 0.087 & .931 & - \\
\hline Victimización & 3.954 & 2.028 & 3.272 & 1.900 & 2.390 & $.019^{* *}$ & .347 \\
\hline
\end{tabular}

\section{DISCUSIÓN}

Ciertamente, la aproximación a la experiencias de violencia en el noviazgo puede hacerse adoptando dos perspectivas, ya sea mediante la información que proporcionan los sujetos sobre su experiencia de victimización (representada, en este caso, por la etiqueta "Maltrato") o la información extraída de los autoinformes, considerando que la respuesta positiva a por lo menos un ítem indica la presencia de una experiencia de victimización en la relación (criterio de tolerancia cero). Tal y como se ha comprobado en este estudio, hay una discrepancia entre ambos criterios ya que mientras que el $3.4 \%$ de los chicos y el $8.4 \%$ de las chicas responden afirmativamente a la etiqueta Maltrato, el $91.4 \%$ de los varones y el $85.2 \%$ de las mujeres han sido víctimas de al menos una conducta abusiva durante su relación. Esta discrepancia parte de la distinción entre maltrato declarado (aquel en el que existe conciencia del abuso) y maltrato técnico (situación en la que están presentes abusos pero no existe conciencia del problema) utilizada por primera vez en la III Macroencuesta Sobre la Violencia contra las Mujeres del Gobierno de España (Instituto de la Mujer, 2006).
Además, en la última Macroencuesta de violencia contra la mujer del Instituto de la Mujer (2015) se prescinde directamente de la etiqueta maltrato y se realizan los análisis en base a las conductas que las encuestadas reportan haber sufrido. Por otra parte, autores como Jouriles et al. (2009) han señalado que, con frecuencia los adolescentes tienden a trivializar los abusos presentes en sus relaciones de pareja, teniendo una conciencia baja del problema. Esta discrepancia se ha comprobado empíricamente utilizando el Cuestionario de Violencia entre Novios (López-Cepero et al., 2015; Rodríguez-Franco et al., 2012) y se ha concluido que por norma general, solo se identifican como maltrato los casos más graves, mientras que las formas más leves de abuso no se tienen en cuenta como tal. En este sentido, en el estudio de Rodríguez-Franco et al. (2016) con dos muestras de mujeres pertenecientes a dos oleadas de evaluación 2003-2005 y 2011 2013 se constata una tolerancia mayor a la experiencia de victimización en las adolescentes y jóvenes participantes en este último periodo. Estos resultados denotan, una vez más, la necesidad de desarrollar e implementar 
estrategias que les permitan identificar correctamente las conductas abusivas, así como las percepciones distorsionadas asociadas a una mayor tolerancia. Por otra parte, el criterio de tolerancia cero indica que la prevalencia de la violencia en el noviazgo es mayor entre los varones (91.4\% frente al $85.2 \%$ de las mujeres), tal y como habían señalado autores como Celis y Rojas (2015) y Cortés-Ayala et al. (2014).

En este estudio, se ha confirmado la existencia de diferencias estadísticamente significativas entre el grupo de víctimas de violencia en el noviazgo y el grupo sobre el que no se ha ejercido violencia, obteniendo los primeros puntuaciones más bajas en autoestima y más elevadas en dependencia emocional. Este hallazgo es refrendado en la literatura sobre el tema (véase Aiquipa, 2015; Pradas y Perles, 2012) y parece indicar que tales modalidades de violencia interpersonal forman parte de un contexto más amplio, tal como habían apuntado Amor et al. (2006) y Póo y Vizcarra (2008), entre otros autores.

Entre los adolescentes victimizados se han hallado diferencias inter-género en dependencia emocional y en autoestima. En su conjunto, siguiendo los hallazgos de López-Cepero et al. (2015), se ha confirmado la presencia de un grado similar de Dependencia Emocional en ambos géneros y de una autoestima más baja en las mujeres, tal y como se esperaba teniendo en cuenta lo señalado por Deza (2012). En este sentido, las adolescentes con experiencias de victimización reportan mayor autoengaño y mecanismos de negación y no afrontamiento y los chicos tienden a la utilización de estrategias de manipulación y acomodación en la relación de pareja. La manipulación se entiende como un intento de modificar los auténticos sentimientos del interlocutor y la acomodación como una adaptación pasiva en la que se deja que sea el otro el que conduzca la relación (Sirvent, 2000). En importante tener en cuenta esto ya que la manipulación podría considerarse un rasgo característico de la Dependencia Emocional Dominante (Pradas y Perles, 2012; Sirvent, 2000). Es decir, esta primera diferencia podría justificarse por la diferencia entre los roles de género entre hombres y mujeres incluso a edades tempranas. Respecto a la acomodación (descrita como el comportamiento de adaptación pasiva al otro/a con falta de iniciativa ante una situación relacional que requeriría de un posicionamiento activo) es importante señalar que en un estudio reciente de Moral et al. (2016), se encontró que las víctimas de abusos en la pareja presentaban acomodación con más intensidad que los que no habían sido victimizados. Por otra parte, Aiquipa (2015) señala que una de las dimensiones más relacionadas con la violencia de la pareja es la subordinación y sumisión, que sería equiparable a lo que nosotros entendemos por acomodación; es decir, la acomodación se acercaría más al patrón conductual de los Dependientes Emocionales Convencionales (Moral y Sirvent, 2009), por lo que podríamos hipotetizar que estas dos formas de Dependencia Emocional coexisten en los varones jóvenes.

Por último, se ha confirmado que los preuniversitarios que han experimentado violencia en el noviazgo presentan una victimización más grave y mayor dependencia emocional en comparación con los universitarios que han experimentado esa misma situación, si bien en autoestima no se han hallado diferencias estadísticamente significativas. Este resultado podría interpretarse aludiendo a la distribución desigual por género y nivel educativo. De este modo, el número de varones participantes en este estudio era superior en el caso de los preuniversitarios por lo que el género puede estar actuando como una variable moduladora de la relación entre nivel educativo, grado de victimización y autoestima ya que se ha hallado que los varones tienen la autoestima más preservada, pese a la experiencia de victimización. En la literatura sobre el tema se confirma un aumento de las experiencias de victimización emocional, así como una mayor frecuencia, en adolescentes de Secundaria en comparación con los universitarios (Blázquez, Moreno y García-Baamonde, 2015; Redondo, Inglés y García, 2017).

Entre las posibles limitaciones de este estudio destaca el uso de un criterio de tolerancia cero que podría limitar los resultados, ya que la muestra se ha dividido en victimizados y no victimizados sin que en este segundo grupo se hayan establecido niveles de gravedad. Aun así, es importante señalar que aunque se han incluido en el grupo de victimizados a todos aquellos que han respondido afirmativamente a un único ítem del CUVINO no se han opacado las relaciones existentes entre la violencia en el noviazgo, la 
dependencia emocional y la autoestima por lo que queda pendiente para trabajos posteriores el estudio de las diferencias entre diferentes tipologías y gravedades de abuso. Ha de hacerse hincapié en que los datos previsiblemente estén sesgados en dirección a la ocultación de casos, tanto por defensividad natural (no consciente) como deliberada (consciente) (véase Arce, Fariña, Seijo y Novo, 2015). Por otro lado, aparte de la posible deseabilidad social, es preciso ser precavidos en la interpretación de los resultados, ya que la extensión total de los cuestionaros aplicados (144 ítems) podría haber provocado fatiga en los participantes.

En cuanto a las implicaciones, es necesario señalar que la violencia en el noviazgo no es una problemática aislada, sino que ha de ser abordada de forma comprehensiva, dados los múltiples factores psicosociales interrelacionados, entre ellos el apego patológico, los procesos de autoengaño, acomodación y manipulación relacional, como principales descriptores de una relación afectivodependiente (Moral et al., 2016). Ello se fundamenta en mecanismos de desigualdad emocional, en opinión de Verdú (2013), factores clave de desregulaciones a nivel afectivo, con mecanismos de sometimiento $y / 0$ sojuzgamiento y juegos de poder, en casos de dependencia emocional. De ahí que se abunde en la necesidad de fomentar modelos de relación afectiva sin desequilibrios de poder (véase Martínez y Rey, 2014) y sensibilizando a edades tempranas, dada la constatación de que ser víctima de violencia en una relación de noviazgo puede actuar como factor de riesgo para victimizaciones posteriores en la adultez (Rodríguez-Franco et al., 2009). Asimismo, se incide en la conveniencia de intervenir sobre las actitudes trascendentes y sexistas, como productos culturales, implicadas en los procesos de distorsión de la detección de las experiencias de victimización en el noviazgo y en la tolerancia al maltrato (Bringas et al., 2017). En este sentido, en el caso de las chicas dependientes emocionales con experiencias de victimización, resultaría de gran relevancia avanzar en propuestas de fortalecimiento de la autoestima como medida encuadrada dentro de intervenciones integrales de carácter multimodal (Moral et al., 2016).

\section{- Conflicto de intereses}

Los autores declaran no tener ningún conflicto de intereses.

\section{REFERENCIAS}

Aguilar, C., Alonso, M. J., Melgar, P. y Molina, S. (2009). Violencia de género en el ámbito universitario: Medidas para su superación. Revista Interuniversitaria de Pedagogía Social, 16, 85-94. https://dx.doi.org/10.7179/ PSRI 2009.16.06

Aiquipa, J. J. (2015). Dependencia emocional en mujeres víctimas de violencia de pareja. Revista de Psicología, 33, 412-437.

Alegría, M. y Rodríguez, A. (2015). Violencia en el noviazgo: Perpetración, victimización y violencia mutua. Una revisión. Actualidades en Psicología, 29 (1 18), 57-72. https://dx.doi. org/10.15517/ap.v29i1 18.16008

Amor, P. y Echeburúa, E. (2010). Claves psicosociales para la permanencia de la víctima en una relación de maltrato. Clínica Contemporánea, 1, 97-104. https://dx.doi. org/10.5093/cc2010v1n2a3

Amor, P., Bohórquez, I. A. y Echeburúa, E. (2006). ¿Por qué y a qué coste psicológico permanece la mujer junto a su pareja maltratadora? Acción Psicológica, 4, 129-154. https://dx.doi.org/10.5944/ap.4.2.483

Arce, R., Fariña, F., Seijo, D. y Novo, M. (2015). Assessing impression management with the MMPI-2 in child custody litigation. Assessment, 22, 769-777. https://dx.doi.org/ 10.1177/1073191114558111

Batiza, F. J. (2017). La violencia de pareja: Un enemigo silencioso. Archivos de Criminología, Seguridad Privada y Criminalística, 18, 144-151.

Blázquez, M., Moreno, J.M. y García-Baamonde, M.E. (2015). Maltrato psicológico en las relaciones de pareja. La inteligencia emocional como factor protector y diferencias de género. Boletín de Psicología, 7 13, 29-47.

Bringas-Molleda, C., Estrada-Pineda, C., Suárez-Álvarez, J., Torres, A., Rodríguez-Díaz, F.J., García-Cueto, E. y Rodríguez-Franco, L. (2017). Actitud sexista y trascendente durante el noviazgo entre universitarios latinoamericanos. Revista Iberoamericana de Psicología y Salud, 8, 44-55. https://doi.org/10.23923/i. rips.2017.08.005

Cala, M.J., Trigo, E. y Saavedra, F.J. (2016). Women's disengagemente from legal proceedings for intimate partner violence sociodemographic and psychosocial variables. The European Journal of Psychology Applied 
to Legal Context, 8, 35-42. https://dx.doi.org/ 10.1016/i.ejpal.2015.10.002

Celis, A. y Rojas, J. (2015). Violencia en el noviazgo desde la perspectiva de varones adolescentes. Informes Psicológicos, 15,83-104. http://dx.doi.org/10.18566/infpsicv15n la05

Cortés-Ayala, L., Bringas-Molleda, C., Rodríguez-Franco, L., Flores-Galaz, M., Ramiro, T. y Rodríguez-Díaz, F. J. (2014). Unperceived dating violence among mexican students. International Journal of Clinical and Health Psychology, 14, 39-47. http://dx.doi. org/10.1016/S1697-2600(14)70035-3

Cortés-Ayala, L., Flores, M., Bringas, C., Rodríguez-Franco, L., López-Cepero, J. y Rodríguez-Díaz, F. J. (2015). Relación de maltrato en el noviazgo de jóvenes mexicanos. Análisis diferencial por sexo y nivel de estudios. Terapia Psicológica, 33, 5-12. http://dx.doi. org/10.4067/s0718-48082015000100001

Cuadrado, I. (2012). Género y rol: Variables que modifican la percepción del maltrato entre iguales. Revista Mexicana de Psicología, 29, 136-146.

Deza, S. (2012). ¿Por qué las mujeres permanecen en relaciones de violencia? Avances en Psicología, 20 (1), 45-55.

Echeburúa, E. (1995). Evaluación y tratamiento de la fobia social. Barcelona: Martínez Roca.

González-Ortega, I., Echeburúa, E. y Corral, P. (2008). Variables significativas en las relaciones violentas en parejas jóvenes: Una revisión. Behavioral Psychology, 16, 207-225.

Hirigoyen, M. F. (2006). Mujeres maltratadas: Los mecanismos de la violencia en la pareja. Barcelona: Paidós Ibérica.

Instituto de la Mujer. (2006). III Macroencuesta Sobre la Violencia contra las Mujeres: Informe de Resultados. Madrid: Ministerio de Trabajo y Asuntos Sociales del Gobierno de España.

Instituto de la Mujer (2015). Macroencuesta de violencia contra la Mujer 2015. Madrid: Ministerio de Sanidad, Servicios Sociales e Igualdad del Gobierno de España.

Jouriles, E. N., Garrido, E., Rosenfield, D. y McDonald, R. (2009). Experiences ofpsychological and physical aggression in adolescent romantic relationships: Links to psychological distress. Child Abuse and Neglect, 33, 451-460. http:// dx.doi.org/10.1016/i.chiabu.2008.11.005

López-Cepero, J., Lana, A., Rodríguez-Franco,
L., Paíno, S. y Rodríguez-Díaz, F. J. (2015). Percepción y etiquetado de la experiencia violenta en las relaciones de noviazgo juvenil. Gaceta Sanitaria, 29, 21-26. http://dx.doi. $\mathrm{org} / 10.1016 /$ i.gaceta.2014.07.006

López-Cepero, J., Rodríguez-Franco, L., Rodríguez-Díaz, F. J., Bringas, C. y Paíno, S. (2015). Percepción de la victimización en el noviazgo de adolescentes y jóvenes españoles. Revista Iberoamericana de Psicología y Salud, 6, 64-71. http://dx.doi.org/10.1016/i. rips.2015.04.001

Martínez, J. A. y Rey, C. A. (2014). Prevención de violencia en el noviazgo: Una revisión de programas publicados entre 1990 y 2012. Pensamiento Psicológico, 12, 117-132. hHp:// dx.doi.org/10.11144/Javerianacali.PPSI12-1. pvnr

Medrano, A., Miranda, M. y Figueras, V. M. (2017). Violencia de pareja contra las mujeres en México: una mirada a la atención del sector salud desde una perspectiva interseccional. Multidisciplinary Journal of Gender Studies, 6, 1231-1262. http://dx.doi.org/10.4471/ generos.2017.2131

Moral, M. V. y Sirvent, C. (2008). Dependencias sentimentales $\mathrm{O}$ afectivas: Etiología, clasificación y evaluación. Revista Española de Drogodependencias, 33, 150167.

Moral, M.V. y Sirvent, C. (2009). Dependencia afectiva y género: Perfil sintomático diferencial en dependientes afectivos españoles. Interamerican Journal of Psychology, 43, 230-240.

Moral, M. V., Sirvent, C., Campomanes, G., Blanco, P., Delgado, R., Quintana, L., Rivas, C., Cuetos, G., Fernández, C. y García, A. (2016). Dependencias relacionales y perpetuación del ciclo de la violencia en adictos. XVII Congreso Virtual Internacional de Psiquiatría - Interpsiquis. Muñoz-Rivas, M. J., Graña, J. L., O'Leary, K.D. y González, M. S. (2007). Aggression in adolescent dating relationships: Prevalence, justification and health consequences. Journal of Adolescent Health, 40, 298-304. http://dx.doi.org/10.1016/i. jadohealth.2006.11.137

Novo, M., Herbón, J. y Amado, B.G. (2016). Género y victimización: efectos en la evaluación de la violencia psicológica sutil y manifiesta, apego adulto y tácticas de resolución de conflictos. Revista Iberoamericana de Psicología 
y Salud, 7, 89-97.http://dx.doi.org/10.1016/i. rips.2016.05.002

Póo, A. M. y Vizcarra, M. B. (2008). Violencia de pareja en jóvenes universitarios. Terapia Psicológica, 26 (1), 84-88. http://dx.doi. org/10.4067/S0718-48082008000100007

Pradas, E. y Perles, F. (2012). Resolución de conflictos de pareja en adolescentes, sexismo y dependencia emocional. Quaderns de Psicología, 14 (1), 45-60.

Redding, E.M., Ruiz, M.T., Fernández, J. y Guijarro, M. (2017). Gender inequality and violence against women in Spain, 2006-2014: towards a civilizited society. Gaceta Sanitaria, 31, 82-88. http://dx.doi.org/10.1016/i. gaceta.2016.07.025

Redondo, J., Inglés, C. J. y García, K. (2017). Papel que juega la edad en el noviazgo de estudiantes de la Universidad Pontificia Bolivariana de Bucaramanga. Diversitas: perspectivas en Psicología, 13 (1), 41 54. Recuperado de: http://revistas.usta. edu.co/index.php/diversitas/article/ download/3491/3381

Rey-Anacona, C. A. (2008). Prevalencia, factores de riesgo y problemáticas asociadas con la violencia en el noviazgo: Una revisión de la literatura. Avances en Psicología Latinoamericana, 26, 227-241.

Rey-Anacona, C. A. (2013). Prevalencia y tipos de maltrato en el noviazgo de adolescentes y adultos jóvenes. Terapia Psicológica, 37, 143-154. http://dx.doi.org/10.4067/S071848082013000200001

Rey-Anacona, C. A., Mateus-Cubide, A.M. y Bayona-Arévalo, P.A. (2010). Malos tratos ejercidos por adolescentes durante el noviazgo: Diferencias por sexo. Revista Mexicana de Psicología, 27, 169-181.

Rodríguez-Franco, L., López-Cepero, J., Rodríguez-Díaz, F. J. y Antuña-Bellerín, M. A. (Junio de 2009). La violencia de género entre parejas de novios universitarios. En I. Vázquez (Coord.), Libro de Actas del / Congreso Universitario Andaluz "Investigación y Género" (pp. 1123-1143). Sevilla: Universidad de Sevilla.

Rodríguez-Franco, L., López-Cepero, J., López, M.I., Paíno, S., Antuña, M.A., Bringas, C. y Rodríguez, F.J. (2016). Evolution of victimization, tolerance and detection of ontimate partner violence among young
Spanish women. Revista de Psicología Social, 37, 137-156. http://dx.doi.org/10.1080/021 34748.2015.1101316

Rodríguez-Franco, L., López-Cepero, J., Rodríguez-Díaz, F.J., Bringas, C., Antuña, M.A. y Estrada, C. (2010). Validación del Cuestionario de Violencia entre Novios (CUVINO) en jóvenes hispanohablantes: análisis de resultados en España, México y Argentina. Anuario de Psicología Clínica y de la Salud, 6, 45-52.

Rodríguez-Franco, L., López-Cepero, J., Rodríguez-Díaz, F. J., Bringas, C., Estrada, C., Antuña, M. A. y Quevedo-Blasco, R. (2012). Labeling dating abuse: Undetected abuse among Spanish adolescents and Young adults. International Journal of Clinical and Health Psychology, 12, 56-67.

Rubio-Garay, F., López-González, M. A., Saúl, L. A. y Sánchez-Elvira-Paniagua, A. (2012). Direccionalidad y expresión de la violencia en las relaciones de noviazgo de los jóvenes. Acción Psicológica, 9, 61-70. hitp://dx.doi. org/10.5944/ap.9.1.437

Sarasua, B., Zubizarreta, I., Echeburúa, E. y Corral, P. (2007). Perfil psicopatológico diferencial de las víctimas de violencia de pareja en función de la edad. Psicothema, 19, 459466.

Sears, H. A., Byers, E. S. y Price, E. L. (2007). The co-occurrence o adolescent boys' and girls' use of psychologically, physically and sexually abusive behaviors. Journal of Adolescence, 30, 487-504. http://dx.doi.org/10.1016/i. adolescence.2006.05.002

Sirvent, C. (2000). Las dependencias relacionales (DR): Dependencia emocional, codependendencia y bidependencia. En Fundación Instituto Spiral (Ed.), Libro del I Symposium Nacional sobre Adicción en la Mujer (pp. 164-215). Madrid: Instituto de la Mujer.

Sirvent, C. (2006). Autoengaño y adicción. Norte de Salud Mental, 26, 39-47.

Sirvent, C. y Moral, M.V. (2005). Inventario de Relaciones Interpersonales y Dependencias Sentimentales I.R.I.D.S-100. Validación de la prueba. Oviedo: Fundación Instituto Spiral y Universidad de Oviedo.

Verdú, A. D. (2013). Género y conflicto en las relaciones de pareja heterosexuales: La desigualdad emocional. Cuestiones de Género: de la lgualdad y la Diferencia, 8, 165-181. http://dx.doi.org/10.18002/cg.v0i8.884 